\title{
A fluidized granular medium as an instance of the Fluctuation Theorem
}

\author{
Klebert Feitosa* and Narayanan Menon ${ }^{\dagger}$ \\ Department of Physics, University of Massachusetts, Amherst, Massachusetts 01003-3720
}

(Dated: October 30, 2018)

\begin{abstract}
We study the statistics of the power flux into a collection of inelastic beads maintained in a fluidized steady-state by external mechanical driving. The power shows large fluctuations, including frequent large negative fluctuations, about its average value. The relative probabilities of positive and negative fluctuations in the power flux are in close accord with the Fluctuation Theorem of Gallavotti and Cohen, even at time scales shorter than those required by the theorem. We also compare an effective temperature that emerges from this analysis to the kinetic granular temperature.

PACS numbers: 05.40.-a, 45.70.-n
\end{abstract}

Take a fistful of marbles in your hand and shake them vigorously. In order to maintain the motions of the marbles, you will have to continuously supply enough energy to them to replenish the energy they lose when they collide inelastically with each other. This is unlike a state of thermal equilibrium, where equal amounts of heat flow into and out of the system of particles to the thermal bath that sets the temperature of the system. All fluctuations in the equilibrium state are given by the appropriate calculation within the canonical ensemble. When this equilibrium state is perturbed slightly (for instance, by connecting the system between two heat baths at slightly different temperatures), the fluctuation-dissipation theorem specifies the linear response of the system in terms of the equilibrium fluctuations. No equivalent framework currently exists for large departures from equilibrium. However, a recent theorem due to Gallavotti and Cohen [1, 2] takes an important step in this direction. Inspired by an observation made in a simulation of a sheared hard-sphere fluid [3], they proved a very general result regarding the entropy flux into a system maintained in a nonequilibrium steady state by a time-reversible thermostat. If dynamics in the system are chaotic [4], then

$$
\Pi\left(\sigma_{\tau}\right) / \Pi\left(-\sigma_{\tau}\right)=\exp \left(\sigma_{\tau} \tau\right)
$$

where $\Pi\left(\sigma_{\tau}\right)$ is the probability of a fluctuation of amplitude $\sigma_{\tau}$ in the rate of entropy production, computed over a time $\tau$ that is much longer than any microscopic time-scale of the system.

The Fluctuation Theorem (FT) embodied in eqn. (10) above, is a remarkably strong statement, with no adjustable system-dependent parameters. The significance and realm of validity of this result are being explored theoretically in various directions. The theorem has been shown to be true under Langevin dynamics [5, 6], thus broadening greatly the range of applicability of systems from only Hamiltonian systems. The theorem has been shown to be equivalent to the fluctuation-dissipation the-

\footnotetext{
*Electronic address: kfeitosa@physics.umass.edu
}

${ }^{\dagger}$ Electronic address: menon@physics.umass.edu orem in the limit of small forces 7]. The FT has also been shown [8] for a chain of anharmonic oscillators to produce the Green-Kubo formula for thermal conductivity. Recent work 9 also shows the connection between the FT and a new thermodynamic identity [10] that relates equilibrium free energy differences to nonequilibrium work. Thus, while eqn. (11) may at first sight appear to be a rather formal mathematical relationship, it has a very nontrivial physical significance.

Explicit realizations of the FT have been demonstrated in simulations of sheared fluids [3], electrical conductivity in an array of fixed scatterers [1], shell-models of turbulence [12], a model of a granular medium and a Burridge-Knopoff type block-spring model [13]. Clear experimental demonstrations of the FT have not previously been achieved, but the two previous attempts have been very instructive in the difficulties involved. Goldburg et al. 14] studied the fluctuations in electrical current required to maintain in steady-state a liquid-crystal film driven into chaotic convectional motion by a constant transverse voltage. No direct test of equation (11) was possible due to the fact that negative fluctuations in power are exceedingly rare in a macroscopic system. Ciliberto and Laroche 15] studied temperature fluctuations in a Rayleigh-Benard cell arguing that the temperature variations at a point in a chaotic flow are representative of the heat fluxes to which the FT applies; they side-step the difficulty of measuring negative power fluctuations encountered in [14 by using as the variable $\sigma$ the local temperature minus the mean temperature in the cell. They find that the ratio on the left side of eqn. (11) is indeed exponential in the amplitude of the fluctuations, but their results are less conclusive on the $\tau$ dependence.

Informed by these previous studies, we are led to consider in this article a granular medium made up of macroscopic spherical beads in which we are able to directly measure energy fluxes. Since granular systems typically contain much fewer particles than molecular systems, and since relevant experimental situations are often strongly driven non-equilibrium states, these fluxes naturally undergo large fluctuations, including the negative fluctuations that play an important role in the FT. These attributes of our experimental system allow us to make a 


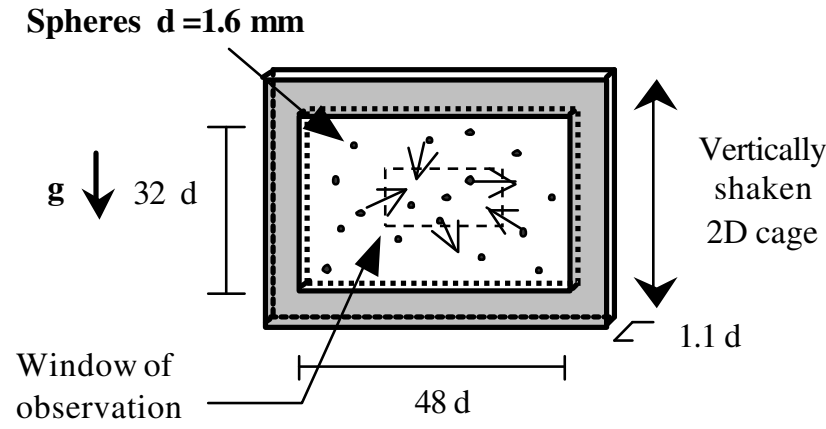

FIG. 1: Sketch of the experimental cell. The dashed rectangle is a window measuring $10 d \times 21 d$, fixed in the laboratory frame, in which we study the flux of kinetic energy.

very substantive test of the result in equation (11).

We make measurements of power fluctuations in the geometry sketched in Figure 11 Spherical glass beads (diameter $d=1.6 \mathrm{~mm}$; mass $m=5.24 \mathrm{mg}$ ) are held in a rectangular cage in the vertical plane and agitated strongly by vertical vibrations. For the measurements we report here, the frequency and amplitude of vibration are held fixed at $60 \mathrm{~Hz}$ and $2.6 d$. We take video frames every $0.5 \mathrm{~ms}$ from which the instantaneous positions of the particles are determined to a precision of $0.025 d$, and their velocities are thence inferred. We measure the time-dependence of the total energy and fluxes into a subsystem defined by the window indicated by dashed lines in Fig. 11 We quantify the power $P(t)$ sustaining the dynamics in this subsystem by finding the sum of two measured quantities. The first is the work done on the particles by gravity, which we obtain from the difference in potential energy of the particles in the window from one frame to the next; this has a zero timeaverage. The second, and much larger, term is the flux in kinetic energy through the dashed lines in Fig. 1 due to particles entering or exiting the window. We neglect the contributions to the $P(t)$ from the flux of rotational kinetic energy. We note that these data are taken in a dilute regime (area fraction $\sim 13.8 \%$ ), so that $P(t)$ is well-approximated by measuring only the contribution from the streaming term, i.e. ignoring the rare contributions to the energy flux from particles at the edges of the subsystem that suffer collisions with particles outside the subsystem.

In the subsystem we consider, the fluctuation over a time $\tau$ of the total mechanical energy is $\Delta E=D+P$, where $D$ is the dissipation due to inelastic collisions between particles, and $P$ is the power driving the subsystem, as described above. In steady state, the timeaverage $\overline{\Delta E}$ is zero, so that $P(t)$ necessarily has a positive time-average value, $\bar{P}$, which must exactly balance the time-average of the dissipation $\bar{D}$. In theoretical discussions of the FT, the entropy production rate, $\sigma$, is identified with the phase space contraction rate. Since this is not an experimentally accessible quantity, we have chosen

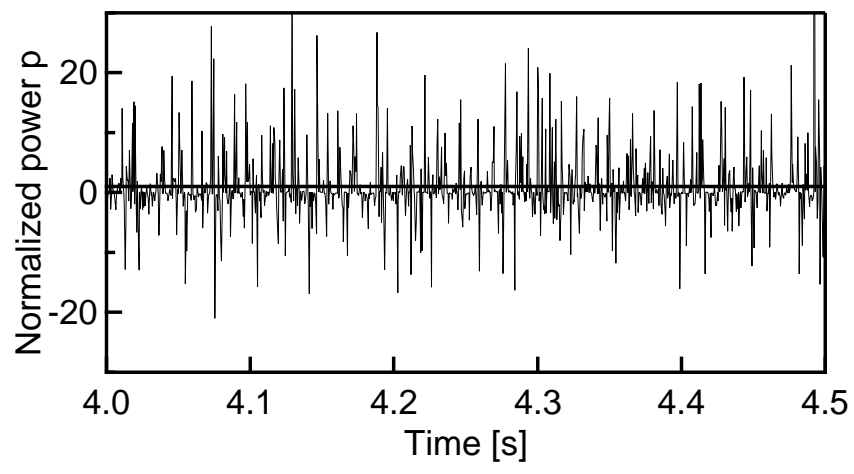

FIG. 2: A sample $(\sim 0.6 \%)$ of the normalized power trace for the subsystem in consideration. The horizontal line shows the average power, $\bar{p}=1$.

to make a correspondence between $\sigma(t)$ and $P(t) / T_{\text {eff }}$, where $T_{\text {eff }}$ is the effective temperature of the system. The choice of what temperature to ascribe to the system is not obvious; we can avoid this issue in constructing the left side of equation (11), since it is a ratio between the probabilities positive and negative values of the same variable. However, we must confront this issue in discussing the right side, since an energy scale will be required to make non-dimensional the quantity in the exponent. We defer this discussion to later in this article.

In Figure 2 we display a sample of the time-variation of the normalized power, $p(t)=P / \bar{P}$ to indicate that this quantity fluctuates strongly about its mean $(\bar{p}=1)$, including making several negative excursions. We emphasize that the occurrence of frequent negative fluctuations is not because we are operating at small driving forces, close to thermal equilibrium; on the contrary, the mean kinetic energy is on the order of $10^{16} k_{B} T$, where $T$ is the ambient temperature that controls the microscopic degrees of freedom internal to the particles.

With $p(t)$ being identified as the variable of interest, the version of the Fluctuation relation that we test here is

$$
\Pi\left(p_{\tau}\right) / \Pi\left(-p_{\tau}\right)=\exp \left(p_{\tau} \tau \bar{P} / T_{e f f}\right) .
$$

Before embarking on the data analysis required to test equation (2), we note a few points that are relevant to the implications of any results that emerge from such an analysis:

- The dynamics in the experimental system are not time-reversible unlike [1, 2] nor do they satisfy microscopic detailed balance unlike the systems of [5, $\underline{6}]$.

- We study an open subsystem of the entire nonequilibrium system rather than the global power injection. The FT is proven for global fluxes and local versions of equation (1) have not been proved except for special cases [16. 


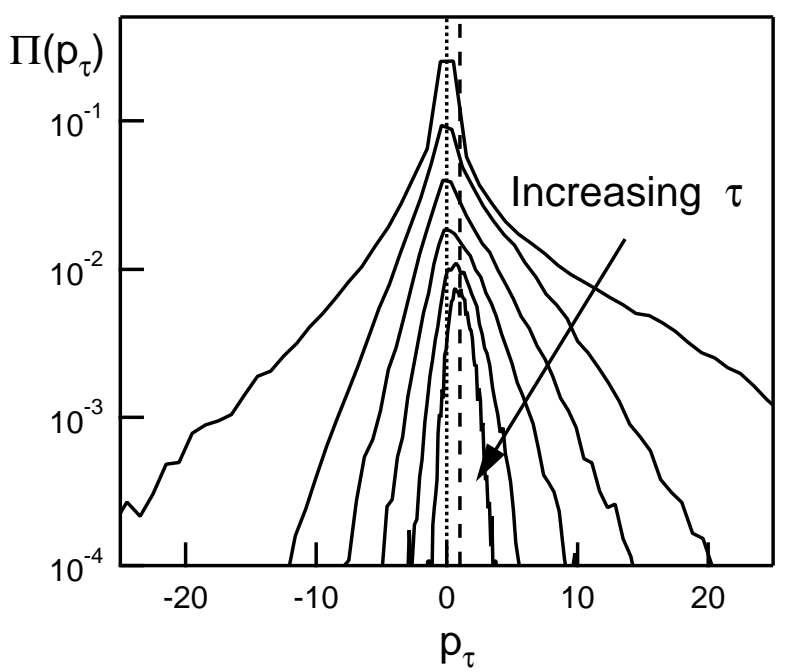

FIG. 3: Probability distribution, $\Pi\left(p_{\tau}\right)$ of the binned power in time bins $\tau=0.5,1,2,4,8$ and $16 \mathrm{~ms}$. The distributions are displaced vertically for clarity. The average power for all distributions is $\bar{p}_{\tau}=1$, indicated by the dashed vertical line.

- To prefigure the data analysis to follow, we find that we are not able to satisfy the condition that $\tau$ is much greater than the dynamical time-scales in the system. However, we find that the statistics of $p(t)$ are strongly non-gaussian for smaller $\tau$ and therefore provide a very demanding test of equation (21).

In order to construct the left side of equation (2), we first partition the time trace of $p(t)$ into non-overlapping bins of duration $\tau$ and compute the average power in these bins, $p_{\tau}(t)=\frac{1}{\tau} \int_{t}^{t+\tau} p\left(t^{\prime}\right) d t^{\prime}$. The probability distribution $\Pi\left(p_{\tau}\right)$ is displayed in figure 3 for $\tau=$ $0.5,1,2,4,8$ and $16 \mathrm{~ms}$ for a data set constructed from a sequence of 170,000 video frames. The data show large, non-gaussian deviations from the mean value of $\bar{p}_{\tau}=1$, with substantial negative tails. The shapes of the distributions vary with $\tau$, thus each distribution provides an independent test of eqn. (2). As noted earlier, even with the large data set that we consider, the values of $\tau$ we are able to access are not truly long compared to the dynamical time-scales of the system, as required by the FT. For comparison, the mean free time is $4 \mathrm{~ms}$ and the time for a particle to diffuse across the window is $10 \mathrm{~ms}$. The autocorrelation function of $p(t)$ shows weak oscillations with a period of $8 \mathrm{~ms}$, which corresponds to the second harmonic of the drive frequency: this is due to energy injection from the top and bottom walls in opposite phases of the drive cycle.

In Fig. $4 \mathrm{~A}$ we show for the probability distributions of Fig. 3. the variation of the ratio $\ln \left[\Pi\left(p_{\tau}\right) / \Pi\left(-p_{\tau}\right)\right]$ with $p_{\tau}$. This ratio is a straight line, as predicted from the exponential dependence in eqn. (11). The only deviations from linearity are the points close to $p=0$ on the $\tau=0.5$

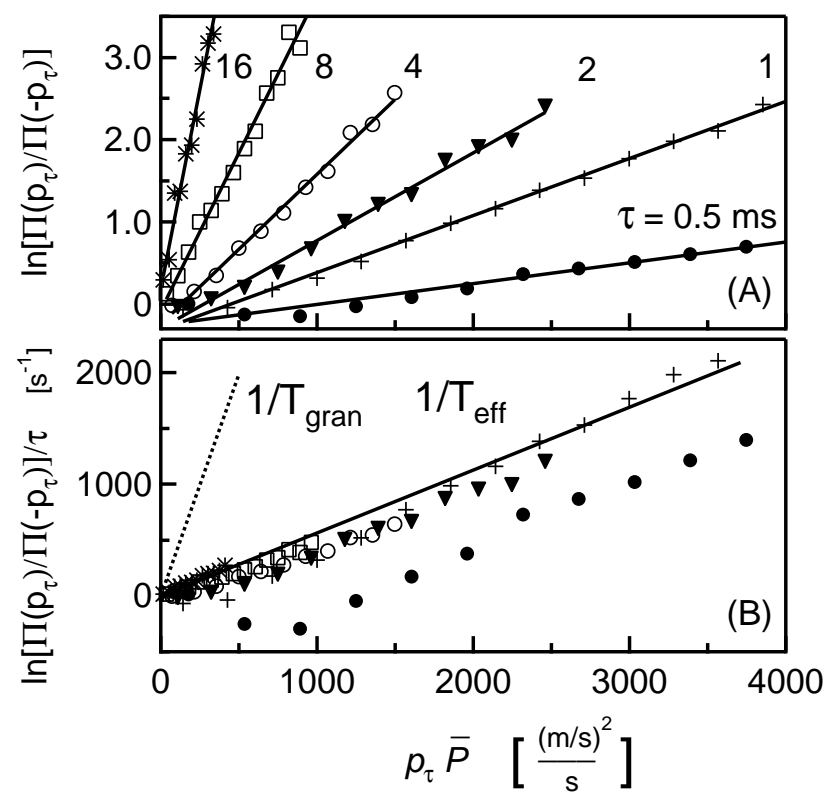

FIG. 4: (A) $\ln \left[\Pi\left(p_{\tau}\right) / \Pi\left(-p_{\tau}\right)\right]$ versus $p_{\tau} \bar{P}$ for $\tau$ ranging from 0.5 to $16 \mathrm{~ms}$. (B) $\ln \left[\Pi\left(p_{\tau}\right) / \Pi\left(-p_{\tau}\right)\right] / \tau$ versus $p_{\tau} \bar{P}$ $\left(\bar{P}=356 \mathrm{~m}^{2} \mathrm{~s}^{-3}\right)$. The solid line shows the slope of the collapsed curves. A dashed line of slope $1 / T_{\text {gran }}$ is drawn for comparison.

and $1 \mathrm{~ms}$ data sets. To study the $\tau$-dependence implied by the FT, in Fig. $4 \mathrm{~B}$ we plot the dependence on $\tau$ of the quantity $\ln \left[\Pi\left(p_{\tau}\right) / \Pi\left(-p_{\tau}\right)\right] / \tau$. For all values of $\tau$, we obtain the same slope (similar results were observed in the simulations in $\operatorname{Ref}[13]$ ), and for large $\tau$, all the data sets collapse on a line passing through the origin, as predicted by eqn. (2). This data collapse indicates a significant degree of agreement with the FT since each of the lines derive from differently shaped $\Pi\left(p_{\tau}\right)$. Furthermore the linear dependence of the ordinate in Figure 4 extends to values of $p_{\tau}$ well beyond the mean so that it seems unlikely that the linearity is merely the leading order behavior in a more complicated functional dependence on $p_{\tau}$.

Finally, we consider the slope of the lines in Fig. [4 as discussed earlier, had we used the rate of entropy flow into the system these lines are predicted to have a universal slope of unity. With our choice of variable, viz.

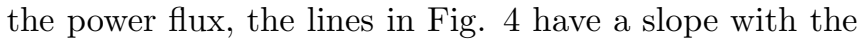
dimension of an inverse energy/mass, which should be given by the inverse of the effective temperature, $T_{\text {eff }}$, of the system. $T_{\text {eff }}$ bears no relation to the ambient temperature. A plausible candidate for a temperature scale, however, is the so-called "granular temperature" $T_{\text {gran }}=1 / 2\left\langle v^{2}\right\rangle$, a purely kinetically defined temperature that we have previously studied 20,21$]$ in the same experimental geometry. The dotted line in Fig. 廿 has a slope of $1 / T_{\text {gran }}: T_{\text {eff }}$ and $T_{\text {gran }}$ are clearly unequal. To study how they are related to one other, in Figure [5] we 


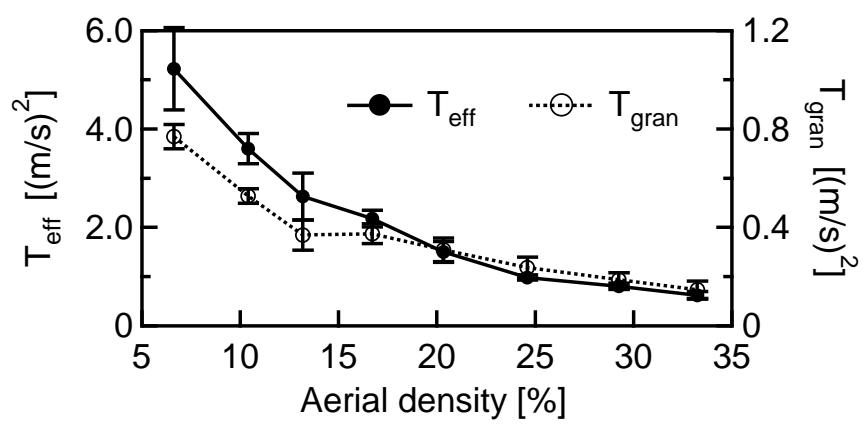

FIG. 5: $T_{\text {eff }}$ (left axis), defined as the inverse of the slope in a graph such as Figure $4 \mathrm{~B}$, and $T_{\text {gran }}$ (right axis), the granular temperature $\left(1 / 2\left\langle v^{2}\right\rangle\right)$, as a function of area fraction. $T_{e f f}$ is numerically larger but follows a similar trend.

display the variation of $T_{\text {eff }}$ and $T_{\text {gran }}$ as a function of the number of particles in the cell, keeping all other parameters of the driving mechanism fixed. As the number of particles increases, $T_{\text {gran }}$ decreases due to an increased rate of inelastic collisions. (We make use of the fact that the slopes of the lines in Fig. 4 do not depend on $\tau$, and plot only the values of the slope for $\tau=1 \mathrm{~ms}$, for which more modest statistics are sufficient). As can be seen, $T_{\text {eff }}$ and $T_{\text {gran }}$ differ in magnitude by about a factor of 8 , however they both decrease similarly as the number of particles is varied.

Prior to the proof of the FT presented in 1, 2], an even stronger statement was proven by Evans and Searles [17] in which they showed that eqn. (1) was true at all $\tau$. This statement, however, applies only to systems that start in equilibrium and are perturbed by a time- independent force. A recent experiment [18] on a colloidal particle pulled through a fluid by an optical trap tests this transient fluctuation theorem by examining an integral version of eqn. (11). The transient fluctuation theorem of Evans and Searles, however, is not expected to apply to the nonequilibrium steady states that we probe here [19, 22]. The fact that eqn. (2) appears to hold at small $\tau$ in our experimental situation should be viewed as a characteristic of the structure of nonequilibrium stationary states that is specific to fluidized granular media.

As discussed earlier, the experimental system is not an idealized instance of the FT: the conditions of timereversibility or detailed balance do not obtain in the experiment, the variable $p(t)$ is not an entropy generation rate, we only study a small, open, subsystem rather than the entire system, and we are not able to go to extremely long timescales. Despite this, we find excellent agreement between our results and the predictions of the FT. This gives hope that these ideas can be extended to some situations where the conditions of proof are not met. The interpretation of the effective temperature in this analysis remains an open question [23]. We speculate that $T_{\text {eff }}$ is potentially valuable in regimes where the granular temperature is no longer expected to be useful.

It is a pleasure to thank O. Narayan, J.S. Urbach, L. Rey-Bellet, and most especially, Giovanni Gallavotti for educative conversations. We also thank O.N. and G.G. for their comments on our manuscript. NM is also grateful to R. Kotecky and other participants at the MPI Workshop on Mathematical Aspects of Material Science who suggested we explore connections of our work with the FT. We received financial support from NSF DMR CAREER 9878433.
[1] G. Gallavotti and E. G. D. Cohen, J. Stat. Phys. 80, 931 (1995).

[2] G. Gallavotti and E. G. D. Cohen, Phys. Rev. Lett. 74, 2694 (1995).

[3] D. J. Evans, E. G. D. Cohen, and G. P. Morriss, Phys. Rev. Lett. 71, 2401 (1993).

[4] More precisely, it must satisfy the Chaotic Hypothesis, which is a defining criterion of an Anosov system, see [1].

[5] J. Kurchan, J. Phys. A 31, 3719 (1998).

[6] J. L. Lebowitz and H. Spohn, J. Stat. Phys. 95, 333 (1999).

[7] G. Gallavotti, J. Stat. Phys. 84, 899 (1996).

[8] L. Rey-Bellet and L. E. Thomas, Ann. Henri Poincare 3, 483 (2002).

[9] G. E. Crooks, Phys. Rev. E 60, 2721 (1999)

[10] C. Jarzynski, Phys. Rev. Lett. 78, 2690 (1997)

[11] F. Bonetto, G. Gallavotti, and P. L. Garrido, Physica D 105, 226 (1997).

[12] L. Biferale, D. Pierotti, and A. Vulpiani, J. of Phys. A,
31, 21 (1998).

[13] S. Aumaitre, S. Fauve, S. McNamara, and P. Poggi, Eur. Phys. J. B 19, 449 (2001).

[14] W. I. Goldburg, Y. Y. Goldschmidt, H. Kellay, Phys. Rev. Lett. 87, 245502 (2001).

[15] S. Ciliberto and C. Laroche, J. Phys. IV 8, 215 (1998)

[16] G. Gallavotti, Physica A 263, 39 (1999)

[17] D. J. Evans and D. J. Searles, Phys. Rev. E 50, 1645 (1994)

[18] G. M. Wang, et al., Phys. Rev. Lett. 89, 050601 (2002)

[19] O. Narayan and A. Dhar, cond-mat/0307148

[20] F. Rouyer and N. Menon, Phys. Rev. Lett. 85, 3676, (2000)

[21] K. Feitosa and N. Menon, Phys. Rev. Lett. 88, 198301 (2002)

[22] R. van Zon, E. G. D. Cohen, Phys. Rev. Lett. 91, 110601 (2003)

[23] G. Gallavotti and E. G. D. Cohen, cond-mat/0312306 